\title{
Prevalence of Germline Brca1 and Brca2 Mutation Among Filipinos
}

\author{
Francisco Tria IV ${ }^{1}$, Daphne Ang ${ }^{1}$, Jose Jasper Andal ${ }^{1}$, Frances Victoria Que ${ }^{2}$, \\ Loraine Kay Cabral $^{3}$, Rosil Dimalibot ${ }^{1}$, Rachelle Arah Salamat ${ }^{1}$, Ma. Luisa Enriquez ${ }^{3}$, \\ Sharlynne Bandales ${ }^{3}$, Raymundo Lo ${ }^{1}$, Manuelito Madrid ${ }^{1}$, Marcelo Imasa ${ }^{3}$, Rubi Li ${ }^{3}$ \\ ${ }^{1}$ Institute of Pathology, St. Luke's Medical Center, Quezon City, Philippines \\ ${ }^{2}$ Department of Medical Oncology, St. Luke's Medical Center, Quezon City, Philippines \\ ${ }^{3}$ Research and Biotechnology Division, St. Luke's Medical Center, Quezon City, Philippines
}

\section{Email address:}

franciscotria@yahoo.com (F. T. IV), daphnechuaang@yahoo.com (D. Ang), josejasperandal@gmail.com (J. J. Andal), ishkaque@yahoo.com (F. V. Que), cabral.loraine@gmail.com(L. K. Cabral), rosildima@gmail.com (R. Dimalibot), rachellearah@gmail.com (R. A. Salamat),ma.luisa.enriquez@dlsu.edu.ph (Ma. L. Enriquez),mvbandales@gmail.com (S. Bandales), raychrislo@gmail.com (R. Lo), migalf22@gmail.com(M. Madrid),marsimasa@yahoo.com (M. Imasa), rubikli@yahoo.com $(\mathrm{R} . \mathrm{Li})$

\section{To cite this article:}

Francisco Tria IV, Daphne Ang, Jose Jasper Andal, Frances Victoria Que, Loraine Kay Cabral, Rosil Dimalibot, Rachelle Arah Salamat, Ma. Luisa Enriquez, Sharlynne Bandales, Raymundo Lo, Manuelito Madrid, Marcelo Imasa, Rubi Li. Prevalence of Germline Brca1 and Brca2 Mutation Among Filipinos. Cancer Research Journal. Vol. 7, No. 3, 2019, pp. 79-86. doi: 10.11648/j.crj.20190703.12

Received: July 6, 2019; Accepted: July 25, 2019; Published: August 10, 2019

\begin{abstract}
The presence of germline mutations in the BRCA1 or BRCA2 tumor suppressor genes are strong predictors of breast or ovarian cancer risk. Loss of the wild-type allele of BRCA1 or BRCA2 genes are required for tumorigenesis. This study identified and characterized the germline BRCA1 and BRCA2 mutation spectrum among Filipinos using Next Generation Sequencing. This is the first local study to perform comprehensive BRCA1 and BRCA 2 (all exons) mutational analysis among Filipinos. This study prompts further investigation of the unique variants to enable better understanding of the genetic predisposition to $\mathrm{BC}$ among Filipinos.
\end{abstract}

Keywords: Hereditary Breast and Ovarian Cancer Syndrome (HBOC), BRCA1 and BRCA2 Genes, Next Generation Sequencing (NGS), Filipino Breast Cancer

\section{Introduction}

The incidence of breast cancer $(\mathrm{BC})$ in Asia is increasing and approximately $10 \%$ of all $\mathrm{BC}$ are hereditary. The most common genetic variations are transmitted in an autosomal dominant manner. Majority of the tested hereditary BC cases show germline mutation in the tumor suppressor genes BRCA1 and BRCA2 conferring a life time risk of $55-85 \%$ and $35-60 \%$ respectively [1].

The presence of germline mutations in the BRCA1 or BRCA2 tumor suppressor genes are strong predictors of developing breast or ovarian cancers. BRCA1 and BRCA2 genes on $17 q 12-21$, and 13q12-13 respectively, are tumor suppressor genes, which function to maintain genomic stability [2]. Mutations in the BRCA gene do not directly result in tumor formation but instead cause genetic instability and trigger further alterations, including inactivation of other tumor suppressor genes and/or activation of oncogenes, leading to malignant transformation [3].

BRCA1 and BRCA2 tumors are associated with different clinico-pathologic and histologic characteristics. Germline BRCA1-associated BC have distinct aggressive features including: early age of onset, high tumor grade, estrogen and progesterone receptor (ER and $\mathrm{PR}$ ) negativity, triple negative breast cancer (TNBC) and high proliferation index. Histologically, BRCA1 positive tumors are high grade invasive ductal carcinomas (IDCA) and more commonly with medullary subtype histology. BRCA1 carriers also have an increased risk of ovarian, prostate, colon, liver and bone cancers. BRCA2-associated BC phenotype are also associated with high grade IDCA, however, it is more 
heterogeneous with age distribution, ER and PR expression, and are not always different from that of sporadic BC. BRCA2 carriers have a lower risk of ovarian cancer when compared with BRCA1 carriers, but they have a higher risk of prostate, pancreatic, gallbladder, pharyngeal, gastric cancers, melanoma, and a higher risk of $\mathrm{BC}$ in men [4-6]. Individuals with a pathogenic variant in BRCA1 and BRCA2 have the following lifetime risk of developing cancers: 40 $80 \%$ for $\mathrm{BC}, 11-40 \%$ for ovarian cancer, $1-10 \%$ for male $\mathrm{BC}$, $1-7 \%$ for pancreatic cancer [7]. In women with ovarian cancer, the presence of BRCA1 or BRCA2 pathogenic variant has been associated with favorable survival rate and longer progression-free survival, after controlling for stage, grade, histology and age at diagnosis [10-11].

The recently approved PARP inhibitor, Olaparib monotherapy, has been shown to reduce the risk of disease progression or death by $42 \%$ and improved progression-free survival by 2.8 months versus standard single-agent chemotherapy in previously treated patients with BRCApositive, Her2-negative BC [8-9]. Olaparib has also been approved in Europe as maintenance in BRCA-mutated, platinum-responsive ovarian cancer patients, and in the USA as a single agent in BRCA-mutated ovarian cancer patients who have received at least three previous chemotherapy lines [12]. Olaparib also received a U.S. Food and Drug Administration breakthrough therapy designation in January 2016 for the treatment of patients with BRCA1 and BRCA2 or ATM gene-mutated advanced castration-resistant prostate cancer (mCRPC) [13].

In this study, we aim to characterize the BRCA1 and BRCA2 mutation spectrum among Filipino patients who met the National Comprehensive Cancer Network (NCCN) criteria of BRCA1 and BRCA2 genetic testing (table 1).

Table 1. National Comprehensive Cancer Network (NCCN) criteria for consideration of BRCA1 and BRCA2 genetic testing.

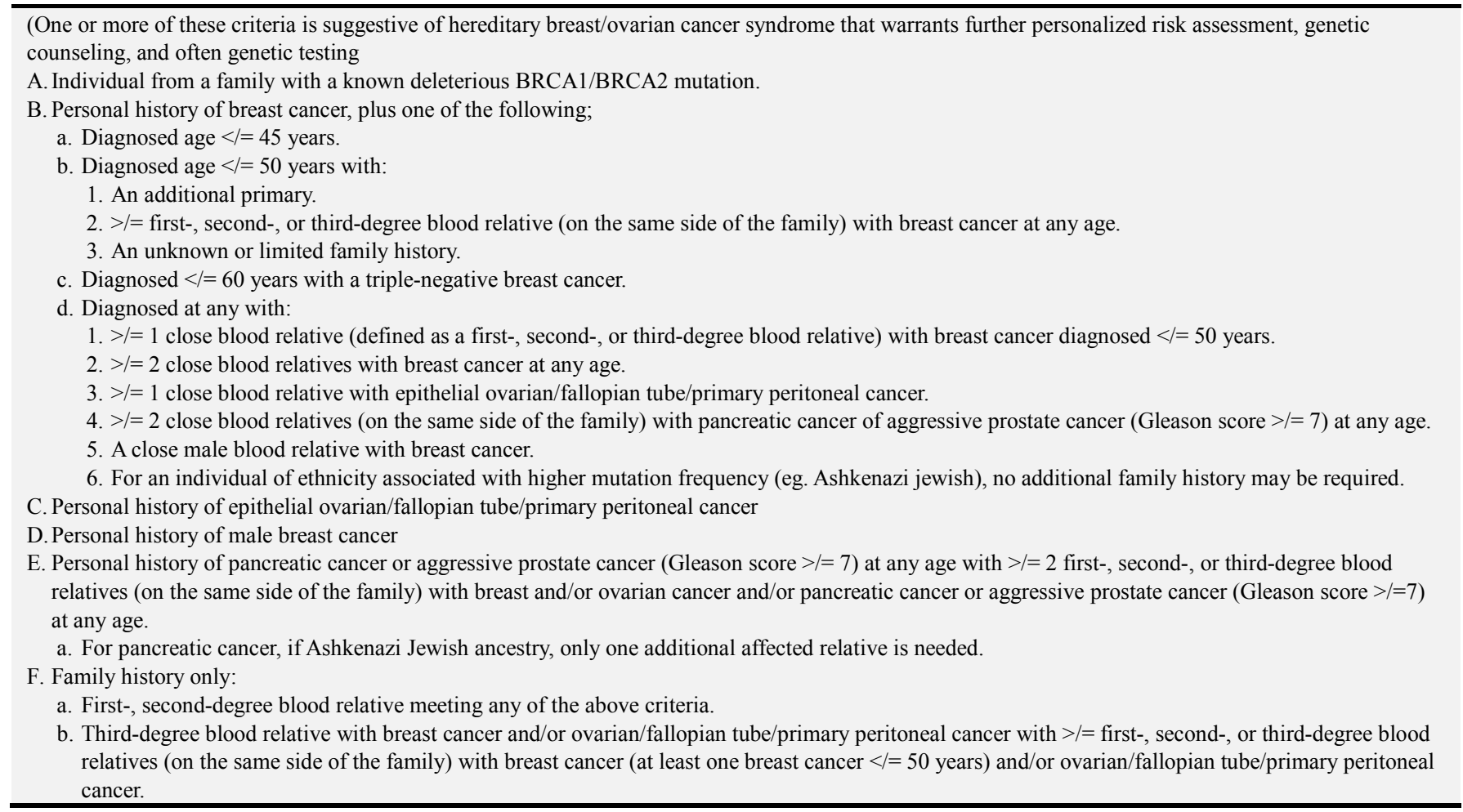

\section{Methodology}

All patients in St. Luke's Medical Center, Quezon City (June 2016-December 2017) who met the National Comprehensive Cancer Network (NCCN) criteria of BRCA1 and BRCA2 genetic testing were included (table 1) [14]. Written informed consent was obtained and pregenetic testing counseling was performed before blood sampling. Clinical and pathologic characteristics (age, sex and diagnosis, family history of cancer, histopathologic diagnosis, and breast hormone receptor panel based on immunohistochemical (IHC) staining results) were recorded (tables 2 and 3 ).

\subsection{NGS Methodology}

Briefly, DNA was extracted from $8 \mathrm{ml}$ of EDTA anticoagulated whole blood, using QiaAmp DNA MiniKit ${ }^{\circledR}$ according to the manufacturer's procedure (Qiagen, USA). The DNA quality was confirmed using a Qubit dsDNA HS Assay kit (Life Technologies, Carlsbad, CA, USA) on a Qubit2.0 Fluorometer (Life Technologies). Library preparation was performed using the Truseq BRCA1 and BRCA2 Custom Amplicon Design-AFP2 according to manufacturer's procedure (Illumina Inc., San Diego, CA, USA). Purification was done using the Agencourt AMPure XP reagent (Beckman Coulter, Brea, CA, USA) and $70 \%$ ethanol on a DynaMagTM-96 Side 
Magnet (Life Technologies). Quality control for the amplicons was established with the High-Sensitivity DNA kit on the Agilent Bioanalyzer (Agilent, Santa Clara, CA, USA). Sequencing was performed using the NGS MiSeq Illumina sequencer. Obtained sequences were aligned to the reference genome (GRCh37hg19) using MiSeq Variant Reporter Software V.3 (Illumina, Inc.). The Miseq VariantStudio v3 data analysis pipeline included base calling, quality score assignment, quality control of mapping quality, coverage analysis, and variant calling. Sequence variants were annotated by BIC Database, ClinVar, and USCS genome browser. Further data review of missense variants was performed by using online software for predicting alterations of protein function, such as SIFT (Sorting Intolerant From Tolerant and PolyPhen-2. Filtering of the data was done manually by reviewing all variants identified by MiSeq Variant Reporter Software V.3 (Illumina, Inc.) Splice site alteration were analyzed $4 \mathrm{bp}$ upstream or downstream of exon-intron boundaries. Sequence data were reviewed with Alamut Visual software and any sequence, alignment, or variant call error artifacts were discarded. Sequence variations were classified as pathogenic, benign, and VUS, based on the most recent 2015 ACMG Standards [15].

All cases with pathogenic BRCA1 and BRCA2 positive mutations, variants of uncertain significance (VUS) calls, and $10 \mathrm{random}$ benign/no pathogenic variants were confirmed by a third-party College of American Pathologists (CAP) accredited molecular laboratory, Veritas Genetics (CLIA ID: 22D2089381), as part of quality control and test validation. The association of the BRCA1 and BRCA2 mutations with tumor histology, grade, hormone status, were determined using Pearson Chi-Square analysis. A p-value of $<0.05$ was considered significant.

\subsection{Hormone Receptor Immunohistochemical Staining}

IHC staining for ER, PR and Her2 neu were performed in Formalin Fixed Paraffin-embedded BC tissue samples, fixed in $10 \%$ neutral-buffered formalin (for 6-8 hrs), using the following antibodies: anti-ER (SP1), anti-PR (1E2), and Her2 (clone 4B5) from Ventana Medical System, Roche (USA). IHCs were performed according to manufacturer's instructions. ER/PR results were determined based on presence of tumor nuclear staining according to Allred Scoring System (table 2). Her2 neu results on the other hand, were determined based on the maximum area of staining intensity, according to the package insert and ASCO/CAP guidelines (table 3 ).

Table 2. ER and PR-ALLRED Score.

\begin{tabular}{llll}
\hline Proportion score (PS) & \% positive cells & Intensity score (IS) & Intensity of positivity \\
\hline 0 & 0 & 0 & None \\
1 & $<1 \%$ & 1 & Weak \\
2 & $1 \%$ to $10 \%$ & 2 & Intermediate \\
3 & $10 \%$ to $33 \%$ & 3 & Strong \\
4 & $33 \%$ to $66 \%$ & & \\
5 & $>66 \%$ & & \\
The PS and IS are added together for a total score: & & Interpretation & \\
Total score (TS) - PS + IS & & Negative & \\
0,2 & Positive & \\
$3,4,5,6,7,8$ & & & \\
\hline
\end{tabular}

Table 3. Her2 neu Immunohistochemical staining scoring.

\begin{tabular}{ll}
\hline Score & Criteria \\
\hline 0 (negative) & No staining is observed or membrane staining that is incomplete and is faint $/$ barely perceptible and in $\leq 10 \%$ of the tumor cells \\
$1+$ (negative) & Incomplete membrane staining that is faint $/$ barely perceptible and in $>10 \%$ of tumor cells \\
$2+$ (equivocal) & weak to moderate complete membrane staining observed in $>10 \%$ of tumor cells \\
$3+$ (positive) & Circumferential membranous staining that is complete, intense and in $>10 \%$ of tumor cells \\
\hline
\end{tabular}

\section{Results}

Among the 192 subjects (age range of 18-76 years old, and mean age of 44 years old), 181 (94\%) were females, and $11(6 \%)$ were males. Ninety-four (94) had BC, 14 with ovarian cancer, 1 with fallopian tube cancer, 1 with primary peritoneal cancer, 4 with concomitant $\mathrm{BC}$ and ovarian cancer, 1 with concomitant $\mathrm{BC}$ and endometrial cancer, and 77 had no cancer at the time of testing.
Thirteen (6.8\%) subjects were identified to have pathogenic BRCA mutations, $10(5.2 \%)$ have VUS, and $169(88.0 \%)$ have benign mutations. The clinicopathologic features and molecular profile of cancer patients enrolled in the study are seen in table 4.

Among patients with BC alone, 9\% (9/99) of the cohort carried the pathogenic BRCA gene.

TNBC were seen in $23 \%(3 / 13), 20 \%(2 / 10)$ and $5 \%$ (9/168) of individuals with BRCA pathogenic mutations, VUS and non-pathogenic group, respectively. 
Table 4. BRCA1/2 status among Filipinos with history of cancer.

\begin{tabular}{|c|c|c|c|c|c|c|c|c|}
\hline \multirow{2}{*}{ Variable } & & & \multicolumn{3}{|l|}{ BRCA1 } & \multicolumn{3}{|l|}{ BRCA2 } \\
\hline & & & Negative & Pathogenic & VUS & Negative & Pathogenic & VUS \\
\hline \multicolumn{3}{|l|}{ No. of subjects $(\mathrm{n}=115)$} & 104 & 6 & 5 & 103 & 7 & 5 \\
\hline \multirow{2}{*}{ Age (years) } & \multicolumn{2}{|l|}{$\leq 45$} & 51 & 5 & 2 & 52 & 4 & 2 \\
\hline & \multirow{2}{*}{\multicolumn{2}{|c|}{$\begin{array}{l}>45 \\
\text { Female }\end{array}$}} & 53 & 1 & 3 & 51 & 3 & 3 \\
\hline \multirow[b]{2}{*}{ Sex } & & & 101 & 6 & 5 & 100 & 7 & 5 \\
\hline & \multicolumn{2}{|l|}{ Male } & 3 & 0 & 0 & 3 & 0 & 0 \\
\hline \multirow{10}{*}{ Family history of cancer } & \multicolumn{2}{|l|}{$\mathrm{BC}$} & 59 & 5 & 3 & 58 & 7 & 2 \\
\hline & \multicolumn{2}{|l|}{ OvCA } & 12 & 2 & 0 & 10 & 3 & 1 \\
\hline & \multicolumn{2}{|l|}{ FTCA } & 3 & 1 & 0 & 2 & 2 & 0 \\
\hline & \multicolumn{2}{|l|}{ Peritoneal CA } & 1 & 0 & 0 & 1 & 0 & 0 \\
\hline & \multicolumn{2}{|l|}{ Pancreatic CA } & 4 & 0 & 1 & 4 & 1 & 0 \\
\hline & \multicolumn{2}{|l|}{ Prostate CA } & 14 & 0 & 0 & 11 & 2 & 1 \\
\hline & \multicolumn{2}{|l|}{ GBM } & 1 & 0 & 0 & 1 & 0 & 0 \\
\hline & $\mathrm{RCC}$ & & 0 & 0 & 1 & 1 & 0 & 0 \\
\hline & $\mathrm{HCC}$ & & 0 & 0 & 1 & 0 & 0 & 0 \\
\hline & None & & 39 & 1 & 1 & 39 & 0 & 2 \\
\hline & & IDCA & 82 & 4 & 3 & 82 & 4 & 3 \\
\hline & & ILCA & 1 & 0 & 0 & 1 & 0 & 0 \\
\hline & $\mathrm{BC} *(n-00)$ & IMCA & 2 & 0 & 0 & 2 & 0 & 0 \\
\hline & BC. $(n-99)$ & IPCA & 0 & 0 & 0 & 0 & 0 & 1 \\
\hline & & DCIS & 6 & 0 & 0 & 5 & 1 & 0 \\
\hline & & Unknown histology & 1 & 0 & 0 & 0 & 0 & 0 \\
\hline & & Mucinous & 0 & 1 & 0 & 1 & 0 & 0 \\
\hline Diagnosis & & Endometrioid & 1 & 0 & 0 & 0 & 0 & 1 \\
\hline & & Clear cell & 6 & 0 & 0 & 5 & 0 & 1 \\
\hline & $\operatorname{OvCA}^{*}(\mathrm{n}=18)$ & Serous & 4 & 0 & 0 & 4 & 0 & 0 \\
\hline & & Germ cell & 1 & 0 & 0 & 1 & 0 & 0 \\
\hline & & Unknown histology & 4 & 0 & 1 & 5 & 0 & 0 \\
\hline & $\operatorname{FTCA}(n=1)$ & & 1 & 0 & 0 & 0 & 1 & 0 \\
\hline & Peritoneal cance & $\mathrm{n}=1)$ & 0 & 0 & 1 & 1 & 0 & 0 \\
\hline & Endometrial can & $*(n=1)$ & 1 & 0 & 0 & 0 & 1 & 0 \\
\hline & $\mathrm{ER}(+)$ & & 60 & 0 & 2 & 56 & 4 & 2 \\
\hline & ER (-) & & 22 & 4 & 1 & 25 & 0 & 2 \\
\hline & Unknown & & 10 & 0 & 0 & 9 & 1 & 0 \\
\hline & $\mathrm{PR}(+)$ & & 55 & 1 & 2 & 52 & 3 & 3 \\
\hline Breact nanel (if with $\mathrm{BC}$ ) & PR (-) & & 25 & 3 & 1 & 27 & 1 & 1 \\
\hline Breast panel (II With BC) & Unknown & & 12 & 0 & 0 & 11 & 1 & 0 \\
\hline & Her2 neu $(+)$ & & 31 & 0 & 1 & 29 & 2 & 1 \\
\hline & Her2 neu (-) & & 51 & 4 & 2 & 52 & 2 & 3 \\
\hline & Unknown & & 10 & 0 & 0 & 9 & 1 & 0 \\
\hline & $\operatorname{TNBC}(n=14)$ & & 10 & 3 & 1 & 13 & 0 & 1 \\
\hline & I & & 21 & 1 & 1 & 22 & 1 & 0 \\
\hline & II & & 33 & 2 & 1 & 33 & 1 & 2 \\
\hline Stage of BC & III & & 19 & 1 & 0 & 16 & 3 & 1 \\
\hline & IV & & 14 & 0 & 1 & 15 & 0 & 0 \\
\hline & Unknown & & 5 & 0 & 0 & 4 & 0 & 1 \\
\hline & I & & 3 & 0 & 1 & 4 & 0 & 0 \\
\hline & II & & 0 & 1 & 0 & 1 & 0 & 0 \\
\hline Stage of OvCA & III & & 2 & 0 & 0 & 2 & 0 & 0 \\
\hline & IV & & 7 & 0 & 0 & 7 & 0 & 0 \\
\hline & Unknown & & 4 & 0 & 0 & 2 & 0 & 2 \\
\hline & 1 & & 1 & 0 & 0 & 0 & 1 & 0 \\
\hline Grade of $\mathrm{BC}$ & 2 & & 7 & 0 & 0 & 7 & 0 & 0 \\
\hline Grade of BC & 3 & & 29 & 3 & 2 & 32 & 2 & 0 \\
\hline & Unknown & & 53 & 2 & 2 & 49 & 4 & 4 \\
\hline
\end{tabular}

CA - cancer; BC - breast cancer; OvCA - ovarian cancer; FTCA - fallopian tube cancer; GBM - glioblastoma multiforme; RCC - renal cell carcinoma; HCC - hepatocellular carcinoma; IDCA - invasive ductal carcinoma; ILCA - invasive lobular carcinoma; IMCA - invasive mucinous carcinoma; IPCA - invasive solid papillary carcinoma; DCIS - ductal carcinoma in-situ; ER - estrogen receptor; PR - progesterone receptor; TNBC - triple negative breast cancer;

* Five (5) patients had double primary carcinomas (4 with concurrent breast and ovarian CA and 1 with concurrent breast and endometrial CA).

\subsection{Patients with BRCA1 and BRCA2 Pathogenic Germline Mutations}

Among the thirteen cases with BRCA1 and BRCA2 pathogenic mutations, $38 \%(5 / 13)$ were identified to be point mutations, including non-sense and missense mutations, $38 \%$ $(5 / 13)$ were insertion/deletion mutations and $23 \%(3 / 13)$ were duplication mutations (tables 5 and 6 ). 
Of the 6 patients with BRCAl pathogenic mutations, 4 $(67 \%)$ patients had breast IDCA, $1(16.5 \%)$ had mucinous ovarian cancer, and $1(16.5 \%)$ was free of cancer at the time of testing. All 4 patients with BRCA1-mutant IDCA were TNBC, and with high Nottingham histologic grade 3 (table $5)$.

Table 5. Characteristics of subjects with BRCA1 pathogenic variants.

\begin{tabular}{llllll}
\hline Subject ID & Variant c & Protein p & Age & Sex & Family history \\
\hline 31 & c. $5398 \mathrm{delC}$ & p.Gln1800AsnfsTer14 & 42 & $\mathrm{~F}$ & BC -1 sister \\
57 & c. $5398 \mathrm{delC}$ & p.Gln1800AsnfsTer14 & 35 & $\mathrm{~F}$ & None \\
90 & c. $4148 \mathrm{C}>\mathrm{G}$ & p.Ser1383Ter & 34 & $\mathrm{~F}$ & BC -1 sister, 1 maternal aunt and 1 paternal aunt \\
113 & c. $7300 \mathrm{G}>\mathrm{A}$ & p.Ser1383Ter & 18 & $\mathrm{~F}$ & BC - mother, 1 maternal aunt and 1 maternal grandaunt; \\
142 & c.5314C $>\mathrm{T}$ & p.Arg1772Ter & 32 & $\mathrm{~F}$ & Ovarian CA - maternal grandaunt \\
172 & c. $2213 \_2214$ maternal grandmother and 3 maternal cousins & BC -2 sisters; & OvCA - maternal cousins \\
\hline
\end{tabular}

Table 5. Continued.

\begin{tabular}{|c|c|c|c|c|c|c|}
\hline Subject ID & Diagnosis & ER & PR & Her2 neu & Grade & Stage \\
\hline 31 & BC, left - IDCA, associated with DCIS & $\mathrm{N}(-)$ & N (-) & $\mathrm{N}(-)$ & NG3, HG3 & IIIA \\
\hline 57 & $\mathrm{BC}$, left - IDCA & N (-) & N (-) & $N(-)$ & HG3, HG3 & IIB \\
\hline 90 & $\mathrm{BC}$, left - IDCA & $N(-)$ & N (-) & $N(-)$ & NG3, HG3 & I \\
\hline 113 & None & $\mathrm{N} / \mathrm{A}$ & N/A & N/A & N/A & N/A \\
\hline 142 & $\mathrm{BC}$, left - IDCA & $\mathrm{N}(-)$ & N (-) & $N(-)$ & NG3, HG3 & IIA \\
\hline 172 & OvCA - mucinous CA & N/A & N/A & $\mathrm{N} / \mathrm{A}$ & $\mathrm{N} / \mathrm{A}$ & IIB \\
\hline
\end{tabular}

CA - cancer; BC - breast cancer; FTCA - fallopian tube cancer; OvCA - ovarian cancer; HG - high grade; IDCA - invasive ductal carcinoma; DCIS - ductal carcinoma in-situ; NG3 - nuclear grade 3; HG3 - histologic grade 3; P (+) - positive; N (-) - negative

Out of the 7 patients with BRCA2 pathogenic mutations, 4 $(58 \%)$ patients had breast IDCA (1 of which has bilateral BC), 1 (14\%) had fallopian tube cancer, 1 (14\%) had concomitant $\mathrm{BC}$ and endometrial cancer, and $1(14 \%)$ was free of cancer at the time of testing (table 6). Lymph node metastasis were identified at diagnosis in $3(23 \%)$ patients with BRCA2 mutations. All 4 BC patients with BRCA2 mutations had high Nottingham histologic grade 3, and were hormone-receptor positive (table 6).

Table 6. Characteristics of subjects with BRCA2 pathogenic variants.

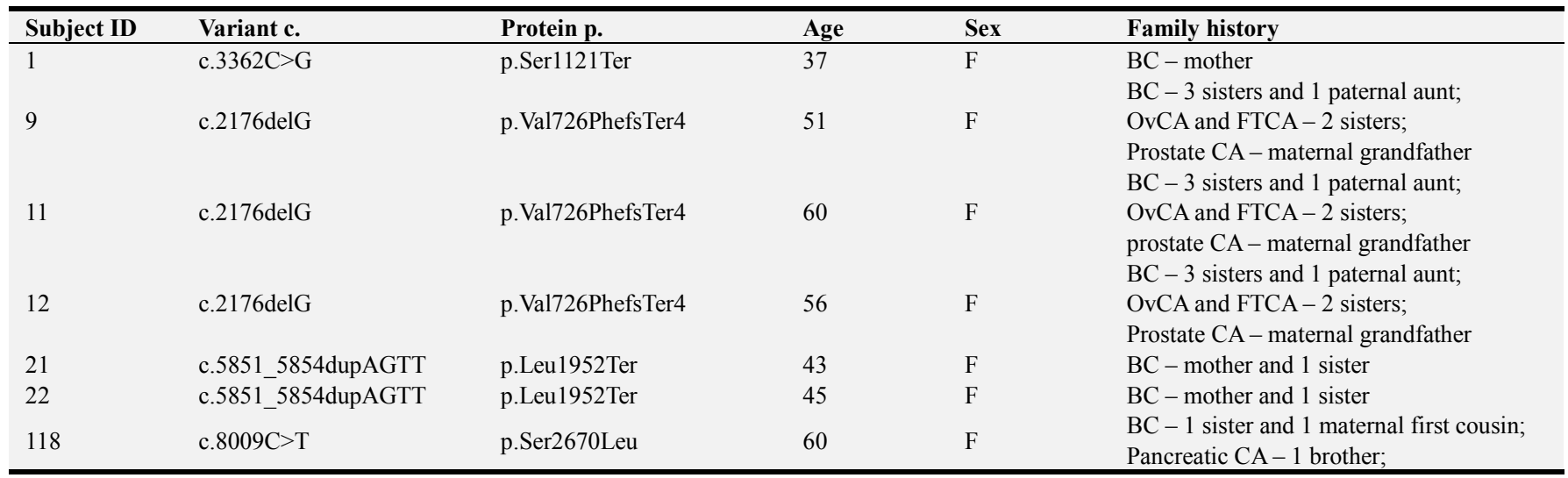

Table 6. Continued.

\begin{tabular}{|c|c|c|c|c|c|c|}
\hline Subject ID & Diagnosis & ER & PR & Her2 neu & Grade & Stage \\
\hline 1 & $\mathrm{BC}$, right - IDCA, with lobular features, associated with HG DCIS & $\mathrm{P}(+)$ & $\mathrm{P}(+)$ & $\mathrm{N}(-)$ & NG3, HG3 & IIIC \\
\hline 9 & $\mathrm{BC}$, right - DCIS with extension to the lobules OvCA & $\mathrm{P}(+)$ & $\mathrm{N}(-)$ & $\mathrm{P}(+)$ & High & I \\
\hline 11 & None & $\mathrm{N} / \mathrm{A}$ & $\mathrm{N} / \mathrm{A}$ & N/A & N/A & N/A \\
\hline 12 & FTCA, bilateral - Serous Papillary CA & N/A & $\mathrm{N} / \mathrm{A}$ & N/A & High & IV \\
\hline 21 & $\mathrm{BC}$, right - IDCA with mucinous features & $\mathrm{P}(+)$ & $\mathrm{P}(+)$ & $\mathrm{N}(-)$ & NG3, HG3 & IIIC \\
\hline 22 & $\mathrm{BC}$ bilateral - IDCA & $\mathrm{P}(+)$ & $\mathrm{P}(+)$ & $\mathrm{N}(-)$ & NG3, HG3 & IIIB \\
\hline 118 & $\begin{array}{l}\mathrm{BC} \text {, right - IDCA; } \\
\text { Endometrial CA - Endometrioid Adenocarcinoma }\end{array}$ & $\mathrm{N} / \mathrm{A}$ & N/A & N/A & HG3, NG3 & IIB \\
\hline
\end{tabular}

CA - cancer; BC - breast cancer; FTCA - fallopian tube cancer; OvCA - ovarian cancer; HG - high grade; IDCA - invasive ductal carcinoma; DCIS - ductal carcinoma in-situ; NG3 - nuclear grade 3; HG3 - histologic grade 3; P (+) - positive; N (-) - negative

BRCA1-mutant patients were significantly more ERnegative than the group without the BRCA1 mutation $(\mathrm{p}=0.02)$.
Although it did not reach statistical significance, BRCA1mutant patients had a higher stage of $\mathrm{BC}$ at diagnosis than 
BRCA1 wild-type patients $(p=0.29)$. On the other hand, the BRCA2-mutant patients had more ER-positive $\mathrm{BC}$ than the BRCA2-negative group ( $p=0.18)$. In contrast to the BRCA1 status, there was no significant difference in the stage at diagnosis of BRCA2-mutant and BRCA2-wild type patients $(p=0.56)$. TNBC was significantly higher in those with pathogenic BRCA mutations than those with BRCA VUS mutations and BRCA wild-type $(p=0.01)$. There was also no significant difference in the age at diagnosis and Her2 neu status in both BRCA1 and BRCA2 mutants versus wild-type patients $(\mathrm{p}=0.41$ and $\mathrm{p}=0.21)$ (table 4$)$.

Among the 77 patients with no cancer at the time of testing, 2 patients had pathogenic BRCA genes (BRCA1
c.7300G $>$ A, p.Ser1383Ter and BRCA2 c.2176delG, p.Val726PhefsTer4).

\subsection{Patients with BRCA1 and BRCA2 VUS Mutations}

Among the $10(5.21 \%)$ cases with VUS, five (50\%) mutations were identified in the BRCA1 gene, and the other five $(50 \%)$ in the BRCA2 gene. Out of the 10 patients with BRCA1 and BRCA2 VUS mutations, 6 had BC (5 IDCA and 1 invasive papillary carcinoma), 1 had ovarian cancer (clear cell carcinoma), 2 had concurrent breast and ovarian cancer, and 1 had primary peritoneal cancer (high grade serous papillary carcinoma). (Refer to Table 7).

Table 7. Characteristics of subjects with Variant of Uncertain Significance (VUS).

\begin{tabular}{|c|c|c|c|c|c|}
\hline Subject ID & Variant c & Protein p & Age & Sex & Family history \\
\hline 7 & c. $824 \mathrm{G}>\mathrm{A}$ & p.Gly275Asp & 28 & $\mathrm{~F}$ & $\mathrm{BC}$ - mother \\
\hline 13 & c. $9104 \mathrm{~A}>\mathrm{G}$ & p.Tyr3035Cys & 57 & $\mathrm{~F}$ & $\mathrm{BC}-$ mother, sister \\
\hline 16 & c. $3308 \mathrm{G}>\mathrm{T}$ & p.Cys 1103Phe & 32 & $\mathrm{~F}$ & N/A \\
\hline 135 & c. $7522 \mathrm{G}>\mathrm{A}$ & p.Gly2508Ser & 48 & $\mathrm{~F}$ & $\mathrm{BC}-1$ aunt, 1 cousin \\
\hline 167 & c. $446 \mathrm{~A}>\mathrm{C}$ & p.Glu149Ala & 56 & $\mathrm{~F}$ & $\mathrm{BC}-$ mother, sister \\
\hline 170 & c. $8515 \mathrm{~T}>\mathrm{C}$ & p.Tyr2839His & 51 & $\mathrm{~F}$ & N/A \\
\hline 182 & c. $824 \mathrm{G}>\mathrm{A}$ & p.Gly275Asp & 58 & $\mathrm{~F}$ & $\begin{array}{l}\mathrm{BC}-2 \text { sisters, mother, grandmother, aunt, cousin; } \\
\text { Pancreatic CA - father }\end{array}$ \\
\hline 184 & c. $5495 \mathrm{C}>\mathrm{A}$ & p.Ser1832Tyr & 44 & $\mathrm{~F}$ & $\begin{array}{l}\text { OvCA - mother; } \\
\text { Prostate } \mathrm{CA}-\text { father }\end{array}$ \\
\hline 189 & c. $.5231 \mathrm{G}>\mathrm{T}$ & p.Ser1744Ile & 31 & $\mathrm{~F}$ & N/A \\
\hline 191 & c. $3596 \mathrm{C}>\mathrm{T}$ & p.Ala1199Val & 66 & $\mathrm{~F}$ & $\begin{array}{l}\mathrm{RCC}-1 \text { brother; } \\
\mathrm{HCC}-1 \text { brother }\end{array}$ \\
\hline
\end{tabular}

Table 7. Continued.

\begin{tabular}{|c|c|c|c|c|c|c|}
\hline Subject ID & Diagnosis & ER & PR & Her2 neu & Grade & Stage \\
\hline 7 & N/A & N/A & N/A & N/A & N/A & $\mathrm{N} / \mathrm{A}$ \\
\hline 13 & $\mathrm{BC}$, right - IPCA & $\mathrm{P}(+)$ & $\mathrm{P}(+)$ & N (-) & NG1, HG1 & N/A \\
\hline 16 & BC, left - IDCA & $\mathrm{P}(+)$ & $\mathrm{P}(+)$ & $\mathrm{P}(+)$ & NG3, HG3 & IV \\
\hline 135 & $\mathrm{BC}$, right - IDCA & N (-) & $\mathrm{N}(-)$ & $N(-)$ & N/A & IIIB \\
\hline 167 & PPCA & N/A & N/A & N/A & N/A & N/A \\
\hline 170 & OvCA - clear cell CA & $\mathrm{N} / \mathrm{A}$ & N/A & N/A & N/A & N/A \\
\hline 182 & $\mathrm{BC}$, right - IDCA & $\mathrm{P}(+)$ & $\mathrm{P}(+)$ & $N(-)$ & NG3, HG3 & II \\
\hline 189 & $\mathrm{BC}$, left - IDCA & $\mathrm{P}(+)$ & $\mathrm{P}(+)$ & N (-) & N/A & IIA \\
\hline 191 & $\mathrm{BC}$, right - IDCA; OvCA, right & $N(-)$ & $\mathrm{N}(-)$ & $N(-)$ & N/A & $\mathrm{N} / \mathrm{A}$ \\
\hline
\end{tabular}

CA - cancer; BC - breast cancer; PPCA - primary peritoneal cancer; OvCA - ovarian cancer; IDCA - invasive ductal carcinoma; RCC - renal cell carcinoma; HCC - hepatocellular carcinoma; NG3 - n HR - hormone receptor; Her2 - Her2 neu; Nuclear grade 3; HG3 - histologic grade 3; P (+) - positive; N (-) negative; N/A - not available

\section{Discussion}

The Philippines has the highest incidence of $\mathrm{BC}$ in Southeast Asia, with a rate of 47.7 per 100,000 per year [16]. One out of 13 Filipino women will develop BC in her lifetime [17]. However, little is known about the inheritance of germline BRCA1 and BRCA2 mutations among Filipinos.

Although less than $5 \%$ of all $\mathrm{BC}$ patients have mutations in the BRCA1 and BRCA2 genes, individuals carrying mutations in either one of these genes have a $47 \%$ to $55 \%$ probability of developing $\mathrm{BC}$, and a $17 \%$ to $39 \%$ risk of ovarian cancer by the age of 70 years [18].

Among our patients who fulfilled the NCCN guidelines (table 1) for BRCA1 and BRCA2 genetic testing, 6.8\% were identified to be pathogenic (3.1\% BRCA1, 3.6\% BRCA2),
$5.2 \%$ were VUS, and $88.0 \%$ were benign (or no pathogenic variant detected). In our patient cohort with previous diagnosis of breast cancer, the incidence of BRCA mutations was $9 \%(4 \%$ in BRCA1 and 5\% in BRCA2). This is slightly lower than the reported prevalence of BRCA1 and BRCA2 pathogenic mutations in Taiwan and China, which are $13.5 \%$ (7.7\% BRCA1, 6.8\% BRCA2) and 18\% (7\% in BRCA1 and $11 \%$ in BRCA2), respectively $[17,19]$. This underscores the need for more comprehensive genetic testing, and looking for alternative genes that may contribute to the development of BC among Filipino patients. The incidence of BRCA2 mutants was also slightly more common than BRCA1 in our study, which is similar to a previous study reported in a Filipino population [16]. Although BRCA1 mutation has been reportedly associated with young age at diagnosis [20], 
it did not reach statistical significance in our study, which may be due to our small cohort size.

Triple-Negative breast cancers were seen in patients with BRCA1 pathogenic mutations and not in BRCA2 mutants. This is similar to a previous report by Peshkin B et al, where they concluded that the proportion of BRCA1 mutations among TNBC cases was always higher $(9-100 \%)$ in BRCA1 vs. BRCA2 (2-12\%) mutations [21].

All BRCA1 mutant cases were negative for ER and Her2 neu by IHC, and are associated with high Nottingham grade. This would have significant treatment implications in terms of it being less responsive to hormone therapy and targeted therapy for Her2 neu [22-24].

There are only few available data of BRCA mutation status among Filipinos. Here, we report 2 unrelated cases with BRCA1 c.5398delC and BRCA2 c.3362C $>\mathrm{G}$ pathogenic mutations (tables 5 and 6), previously described in the Filipino population [16]. A case of 2 Filipino family members (siblings) from our study, showed a BRCA2 c.5851_5854dupAGTT mutation, previously described in the Chinese and Indian populations, but not yet reported among Filipinos. Another Filipino patient with a concomitant breast and endometrial cancer showed the pathogenic BRCA2 c. $8009 \mathrm{C}>\mathrm{T}$ mutation (table 6), previously reported in the Japanese, also not reported yet in Filipinos [16]. The Filipino population is one of the most diverse ethnic groups in the world, and this probably contributes to the diverse genotypic make-up of the Filipino ancestry [25-26].

This has been the first local study in the Philippines to use NGS platform for BRCA1 and BRCA2 testing among Filipinos. Benefits of BRCA1 and BRCA2 mutation testing include optimal treatment tailored for BRCA-positive patients and, more importantly, extending testing to family members and helping them take measures for early detection of cancer. More comprehensive surveillance and clinical follow-up, including radiological tests and more frequent physician consults, may be advised to those patients that show pathogenic mutations in their BRCA genes. Aside from the BRCA1 and BRCA2 genes, testing germline mutations of other genes related to breast and ovarian cancer, will be helpful if high suspicion of a hereditary disease is suspected. Perhaps one limiting factor for Filipinos to be tested would be the prohibitive cost of each special test. Cost-effective analysis and financial support are needed to further extend genetic testing to an average Filipino individual.

\section{Conclusion}

In this study, we were able to demonstrate that pathogenic mutations in the BRCA genes were seen in $6.8 \%$ of Filipinos who fulfilled the NCCN criteria for BRCA1 and BRCA2 genetic testing. The incidence of BRCA2 mutant genes were slightly more frequent than BRCA1 (3.6\% vs. 3.1\%). We also report that all TNBC were seen in individuals with pathogenic BRCA1 mutations, while hormone-receptor positive $\mathrm{BC}$ cases were seen in individuals with pathogenic BRCA2 mutations. Although BRCA1 and BRCA2 genes contribute to a large percentage of patients with $\mathrm{HBOC}$, several other susceptible genes need to be further analyzed, to identify other genes associated with hereditary cancer syndromes among Filipinos.

\section{Limitations of the Study}

Large deletions/duplications were not detected due to inherent limitations of NGS. These can be further detected using a separate technology such as Multiplex Ligationdependent Probe Amplification (MLPA) analysis. Other genes associated with HBOC (such as CHEK2, ATM, and PALB2) also need to be analyzed to identify other hereditary syndromes associated with $\mathrm{BC}$ and ovarian cancer.

Another limitation in our study is the inherent higher prevalence of $\mathrm{BC}$ among Filipino patients over ovarian cancer and other BRCA-associated malignancies. Thus, majority of the patients enrolled in this study were BC patients. Clinical data on treatment response and/or progression-free survival were also not available for analysis in this current study.

\section{Ethical Consideration}

This study was approved by the Institutional Review Board (IRB) and the Institutional Ethics Review Committee (IERC) of the St. Luke's Medical Center. The BRCA1/2 mutation status/information was only available to the patient. Any information that identifies the patient to anyone without the patient's permission were not divulged. The researchers who studied the sample were not able to identify the patient.

\section{Authors Contribution}

Francisco P. Tria IV, MD - First author, Manuscript writing, Data collation (Pathologist)

Daphne C. Ang, MD - Corresponding author, interpretation of molecular data, manuscript writing (Pathologist)

Jose Jasper L. Andal, MD - Interpretation of molecular data (Pathologist)

Frances Victoria Que, MD - Patient recruitment, Patient counselling (Medical Oncologist)

Loraine Kay D. Cabral, MSc - Technical staff, Patient counselling

Rosil Dimalibot, RMT - Technical staff

Rachelle Arah Salamat, RMT - Technical staff

Sharlynne Bandales, RN - Project assistant

Ma. Luisa D. Enriquez, PhD - Consultant staff

Raymundo W. Lo, MD - Biopsy interpretation

(Pathologist)

Manuelito A. Madrid, MD - Biopsy interpretation (Pathologist)

Marcelo Imasa, MD - Consultant staff

Rubi K. Li, MD - Senior consultant (Medical Oncologist)

\section{Consent for Publication}

The authors, subjects, and funding authorities had consented 
that this research be published in an international journal.

\section{Competing Interests}

The authors declared that they have no competing interests.

\section{Availability of Data and Material}

The data gathered from this research was made available to the subjects and attending physicians of patients involved in the study. The raw data and materials were kept in a secured place at St. Luke's Medical Center.

\section{Acknowledgements}

This research was funded by AstraZeneca Philippines, and St. Luke's Medical Center Research and Biotechnology Division.

\section{References}

[1] Her2 neu Learning about the BRCAX study. Genome. gov. National Research Genome Institute (NIH). Available: http://www.genome.gov/10000532.Accessed 2015 April 20.

[2] Her2 neu Boulton SJ. Cellular functions of the BRCA tumoursuppressor proteins. Biochem. Soc. Trans. (Pt 5): 633-45. November 2006.

[3] Her2 neu Chong HK, Tang T, Lu HM, et al. The Validation and Clinical Implementation of BRCAplus: A Comprehensive HighRisk Breast Cancer Diagnostic Assay. PLoS ONE 9 (5): e97408. https://doi.org/10.1371/journal.pone.0097408. May 2014.

[4] Her2 neu Cheng L, Eble J, et al. Molecular Pathology of Breast Cancer. Springer Science + Business Media New York. 2013.

[5] Her2 neu Greer J, Whitcomb D. Role of BRCA1 and BRCA2 mutations in pancreatic cancer. Gut. 10. 1136/gut. 2006. 101220. 2007.

[6] Her2 neu Naderi A, Couch F. BRCA2 and pancreatic cancer. Int J Gastrointest Cancer. 31 (1-3): 99-106. 2002.

[7] Her2 neu Cavanagh H, Rogers K. The role of BRCA1 and BRCA2 mutations in prostate, pancreatic and stomach cancers. Hereditary Cancer in Clinical Practice. ISSN: 1897-4287. 2015.

[8] Her2 neu Alsop K, Fereday S, Meldrum C, et al. BRCA mutation frequency and patterns of treatment response in BRCA mutation-positive women with ovarian cancer: a report from the Australian Ovarian Cancer Study Group. J Clin Oncol. 2012; 30: 2654-63. 2012.

[9] Her2 neu Robson M, Im SA, Senkus E, et al. Olaparib for metastatic breast cancer in patients with a germline BRCA mutation. 2017; 377: 523-33. N Engl J Med. doi: 10. 1056/NEJMoa1706450. 2017.

[10] Her2 neu Andei AZ., Zogopoulos G., Pelletier. J., et al. Therapeutic approaches to BRCA2-associated pancreatic cancer. Experimental Medicine, McGill University, Montreal. 2015.

[11] Her2 neu Bolton KL, Chenevix-Trench G, Goh C, et al. Association between BRCA1 and BRCA2 mutations and survival in women with invasive epithelial ovarian cancer. JAMA. 2012; 307: 382-90. 2012.

[12] Her2 neu Martin GA, Chen AH, Parikh K. A Novel Use of Olaparib for the Treatment of Metastatic Castration-Recurrent Prostate Cancer. Pharmacotherapy. 2017 Nov; 37 (11): 14061414. doi: 10. 1002/phar. 2027. 2017.

[13] Her2 neu Slomski A. Olaparib Increased Survival in Metastatic Breast Cancer. Clinical Trials Update. August 22/29, 2017.

[14] Her2 neu NCCN Guidelines Version 1. 2018. BRCA-Related Breast and/or Ovarian Cancer Syndrome. 10/03/17 (C) National Comprehensive Cancer Network, Inc. 2017.

[15] Her2 neu Richards S, Aziz N, Bale S, et al. Standards and Guidelines for the Interpretation of Sequence Variants: A Joint Consensus Recommendation of the American College of Medical Genetics and Genomics and the Association for Molecular Pathology. Genet Med. 2015 May; 405-424. Published online 2015 Mar 5. doi: 10. 1038/gim. 2015. 30.

[16] Her2 neu De Leon Matsuda ML, Liede A, Kwan E, et al. BRCA1 and BRCA2 mutations among breast cancer patients from the Philippines. Int. J. Cancer: 98, 596-603. 2002.

[17] Her2 neu http://www.pbcn.org/index.html

[18] Her2 neu Sung P-L, Wen K-C, Chen Y-J et al. The frequency of cancer predisposition gene mutations in hereditary breast and ovarian cancer patients in Taiwan: From BRCA1/2 to multi-gene panels. PLoS ONE 12 (9): e0185615. https://doi.org/10.1371/journal.pone.0185615.2017.

[19] Her2 neu Yang $\mathrm{X}, \mathrm{Wu} \mathrm{J}, \mathrm{Lu} \mathrm{J}$ et al. Identification of a Comprehensive Spectrum of Genetic Factors for Hereditary Breast Cancer in a Chinese Population by Next- Generation Sequencing. PLoS ONE 10 (4): e0125571. doi: 10. 1371/journal. pone. 0125571. 2015.

[20] Her2 neu Kwong A, Shin V, Wo J, et al. Comprehensive spectrum of BRCA1 and BRCA2 deleterious mutations in breast cancer in Asian countries. J Med Genet 2016; 53: 1523. doi: 10. 1136/jmedgenet-2015-103132. 2016.

[21] Her2 neu Peshkin B., Alabek M., Isaacs C. BRCA1/2 Mutations and Triple Negative Breast Cancers. NIH Public Access. Breast Dis. 2010; 32 (0): doi: 10. 3233/BD-2010-0306. 2013.

[22] Her2 neu Eisinger F., Lyonnet DS, Longy M, et al. Germ line mutation at BRCA1 affects the histoprognostic grade in hereditary breast cancer. Cancer Res 1996; 56: 471-4, 1996.

[23] Her2 neu Marcus JN, Page DL, Watson P, et al. BRCA1 and BRCA2 hereditary breast carcinoma phenotypes. Cancer 1997; 80: 43-56. 1997.

[24] Her2 neu Johannsson TO, Idvall I, Anderson C, et al. Tumor biological features of BRCA1-induced breast and ovarian cancer. Eur J Cancer 1997; 33: 362-71. 1997.

[25] Her2 neu Chu R. Reconstituting Histories of Filipino Families with Chinese Ancestry: Methodology, Sources, and Relevance. Kritika Kultura 21/22 (2013/2014): -029. (C) Ateneo de Manila University. $<$ http://kritikakultura.ateneo.net $>$

[26] Her2 neu Mulder N. Filipino Identity: The Haunting Question. Journal of Current Southeast Asian Affairs, 32, 1, 55-80. ISSN: 1868-4882 (online), ISSN: 1868-1034 (print). 